\title{
Effects of parasympathetic denervation of the salivary glands on feeding and drinking behavior in the rat
}

\author{
JUAN M. J. RAMOS and AMADEO PUERTO \\ Laboratorio de Psicobiologia, Universidad de Granada, Granada, Spain
}

\begin{abstract}
The present experiment was undertaken to study the function of parasympathetic innervation of the salivary glands on food-associated drinking in the rat. Several studies have shown nonhomeostatic polydipsia and a high number of drinking responses in the presence of dry food after removal of the salivary glands. The neural mechanisms responsible for this phenomenon, however, have not been investigated. This study shows that transection of the preganglionic parasympathetic salivary fibers at the point where they traverse the middle ear in the rat led to the development of a prandial drinking pattern. As a secondary effect of prandial drinking, experimental animals ingested a large volume of water in the presence of dry food; that is, significant polydipsia was elicited. Both the increased frequency of drinking and polydipsia were reversed when the regular dry food was replaced by a wet diet. These results are discussed in relation to the possible connection between parasympathetic innervation of the salivary glands and the prandial drinking pattern characteristically observed in desalivated rats.
\end{abstract}

It is commonly accepted that saliva plays an important role in feeding behavior (Powley, 1977). From a behavioral standpoint, the deficit in salivary secretion induced by removal of the three major salivary glands (submandibular, sublingual, and parotid) in the rat is followed by alternating sequences of the eating of small pieces of dry food and the drinking of minute amounts of water, leading to a significant increase in drinking frequency (Stricker, 1970). This characteristic prandial pattern of drinking also produces a sustained increase in nonhomeostatic or secondary water intake while dry food is being consumed (Epstein, Spector, Samman, \& Goldblum, 1964). This peculiar and consistent change in feeding habits ("prandial drinking") results from the use of water as an exogenous saliva to facilitate the swallowing of dry food (Kissileff, 1969).

Although these studies recorded prandial drinking following salivary gland removal, the neural mechanisms mediating this phenomenon have not yet been investigated. In the rat, the salivary glands are doubly innervated; hence, parasympathetic and sympathetic saliva can be secreted via stimulation of the chorda tympani/tympanic nerve or the superior cervical ganglion, respectively (Emmelin, 1987; Thulin, 1976). However, the saliva

\footnotetext{
This report is based on a portion of a dissertation submitted in partial fulfiliment of the requirements for the PhD degree, University of Granada, Spain (University Microfilms No. 84-338-0575-4-J. M. J. Ramos, 1987). We thank Karen Shashok for assisting with the translation of the original manuscript into English. Correspondence should be addressed to Juan M. J. Ramos, Laboratorio de Psicobiología, Area de Psicobiología, Campus de Cartuja, Universidad de Granada, Granada, Spain E-18071.
}

secreted after stimulation of the sympathetic nerves is highly viscous (i.e., rich in electrolytes and poor in water), whereas parasympathetic saliva is watery, and the lack of parasympathetic saliva leads to a high degree of mouth dryness (Abe, 1987; Abe \& Nitta, 1984). These findings suggest that parasympathetic control of the salivary glands may play a significant role in prandial drinking and nonhomeostatic polydipsia. The aim of the present study was, therefore, to analyze the changes in the feeding and drinking habits of rats after denervation to determine whether parasympathetic secretory input to the salivary glands is responsible for the prandial style of drinking seen in desalivated animals.

\section{METHOD}

\section{Subjects}

A total of 16 Wistar rats (240-370 g) were randomly assigned to either an experimental $(n=8)$ or a control group $(n=8)$. Animals were housed in individual cages with food and water ad lib, except where indicated otherwise. Room temperature was kept at $20-22^{\circ} \mathrm{C}$ and a constant $12: 12-\mathrm{h}$ light:dark cycle was used (lights on at $0800 \mathrm{~h}$ ).

\section{Surgical Procedure}

Salivatory fibers of both the chorda tympani and the tympanic nerve, that is, the preganglionic parasympathetic fibers to the submandibular-sublingual and parotid glands, respectively, pass through the tympanic cavity of the middle ear (Nicholson \& Severin, 1981; Nomura \& Mizuno, 1982). We took advantage of this anatomical proximity to section both nerves simultaneously at this level. In the experimental group, bilateral sectioning of the chorda tympani and tympanic nerve was performed under sodium thiopental anesthesia ( $42 \mathrm{mg} / \mathrm{kg}$, i.p.) according to a slight modification of a technique developed by other authors (Nicholson \& Severin, 1981; Stricker, 1970). A blunt metal probe was inserted into the external 
auditory meatus until the probe reached the middle ear, lesioning the salivatory fibers. The same procedure was used in control animals, except that the blunt metal probe was inserted to a location short of the middle ear.

\section{Testing Procedure}

After surgery, the animals were allowed 10 days to recover with water and Purina Lab Chow pellets available ad lib (day of surgery was Day 14). At the end of the recovery period, both control and experimental rats were habituated over a period of 7 days to eating dry food from 1000 to $1200 \mathrm{~h}$ only; water remained available ad lib. Over the next 3 days (Days 32, 33, and 34), the number of drinking responses emitted by all subjects was recorded during the 2-h period when dry food was presented. On Days 35, 36, and 37 , a wet mash diet consisting of 3 parts isotonic saline to 2 parts dry diet was substituted for the dry food, and the number of drinking responses was also recorded throughout the 2 -h feeding period. Finally, on Day 38, the study was concluded by presenting the regular dry food during the 2-h feeding period and recording the frequency of drinking episodes. The number of drinking responses emitted by both the experimental and control rats throughout the experiment was visually assessed by counting each approach to the drinking spout and subsequent consumption of water, as demonstrated by the appearance of air bubbles rising from the spout as the animal swallowed.

The amount of food ingested and the amount of water drunk by all subjects were also recorded during the 2-h feeding period on all seven test days (from Day 32 to Day 38 ). Water drunk during the 22-h period of food deprivation was also measured.

\section{Statistical Analysis \\ Mixed ANOVAS (section $\times$ days) and Student's $t$ test for in- dependent samples were used where appropriate.}

\section{RESULTS}

\section{Prandial Drinking}

Baseline results are shown in Figure 1. During the baseline period (Days 8 to 14), the experimental and control groups did not differ significantly in the number of drinking responses when they were presented with dry food $[F(1,14)=1.822$, n.s. $]$ or wet food $[F(1,14)=0.781$, n.s.]. However, after transection of the parasympathetic salivatory fibers, the frequency of drinking observed on Days 32,33 , and 34 in the presence of dry food was significantly higher in experimental animals than it was in the control group $[F(1,14)=80.706, p<.001$; Figure 1]. These differences were significant on Days 32 $[t(14)=8.371, p<.001], 33[t(14)=9.914, p<$ $.001]$, and $34[t(14)=7.910, p<.001]$.

On Days 35, 36, and 37, the presentation of the wet mash diet reversed the prandial style of drinking previously observed in subjects with middle ear lesions $[F(1,14)$ $=1.703$, n.s.; Figure 1]. However, when dry food was re-introduced on Day 38, animals with middle ear lesions resumed a prandial drinking pattern, with increased frequency of drinking $[t(14)=8.282, p<.001]$.

\section{Water Intake}

As a result of the dramatic rise in the number of drinking responses observed during the 2-h period when dry food was presented, the experimental subjects consumed twice the volume of water as did the controls, showing

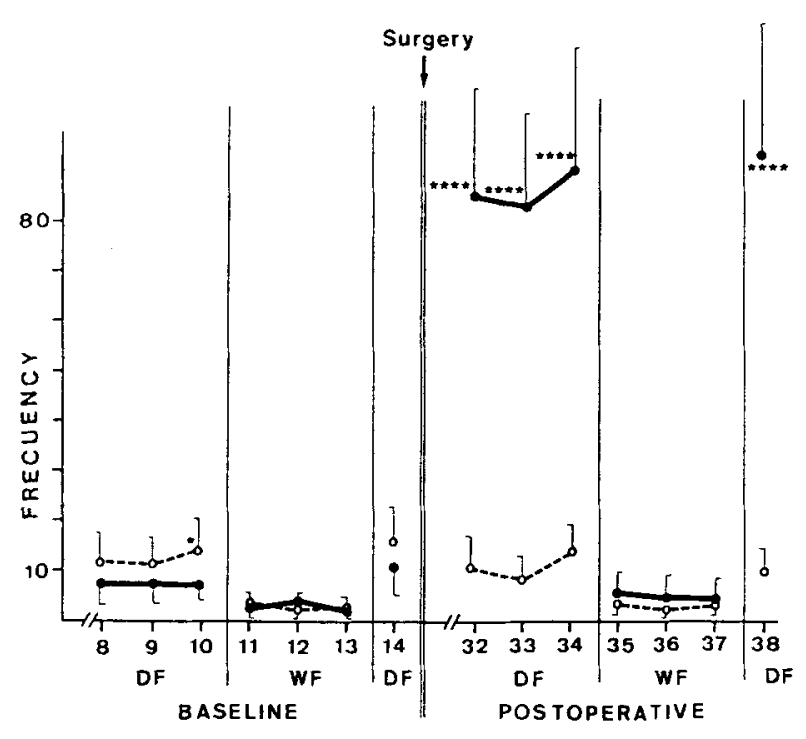

\section{A Y S}

Figure 1. Mean frequency $( \pm S D)$ in experimental (solid line) and control (dotted line) rats during baseline (Days 8-14) and experimental postoperative periods (Days 32-38). DF = dry food; WF = wet food. Significance level: ${ }^{*} p<.05,{ }^{* *} p<.025,{ }^{* * *} p<.01$, ${ }^{* * * *} p<.001$. Statistical comparisons were performed with Student's $t$ test. Surgery was on Day 14.

a clear polydipsic effect. Following transection of the salivatory nerves, the experimental rats drank significantly larger amounts of water than did the control animals during the 2-h period of exposure to dry food on Days 32, 33 , and $34[F(1,14)=20.979, p<.001$; Figure 2]. These differences were significant on Days $32[t(14)=$ $4.768, p<.001], 33[t(14)=4.538, p<.001]$, and $34[t(14)=3.749, p<.01]$. On Days 35, 36, and 37, the presentation of the wet mash diet abolished the polydipsia induced by transection of the parasympathetic fibers, as shown by the finding that experimental subjects consumed a volume of water similar to that recorded for the control group $[F(1,14)=0.016$, n.s.; Figure 2]. When dry food was re-introduced on Day 38 , the experimental rats once again exhibited polydipsia, ingesting a significantly larger volume of water than did the control animals $[t(14)=3.705, p<.01]$.

Despite the polydipsia observed in the experimental group during the 2-h period when dry food was offered (Days 32, 33, and 34), the experimental rats showed hypodipsia throughout the remaining $22 \mathrm{~h}$ of daily food deprivation, in comparison with the control rats. During the 22-h period of food deprivation, the experimental group drank a significantly lower volume of water than did the controls $[F(1,14)=9.492, p<.01$; Figure 3]. However, during the $22 \mathrm{~h}$ subsequent to the wet mash diet (Days 35, 36, and 37), the experimental and the control rats drank statistically similar amounts of water $[F(1,14)$ $=.030$, n.s.; Figure 3]. Both groups consumed similar amounts of food during the postoperative phase of the 


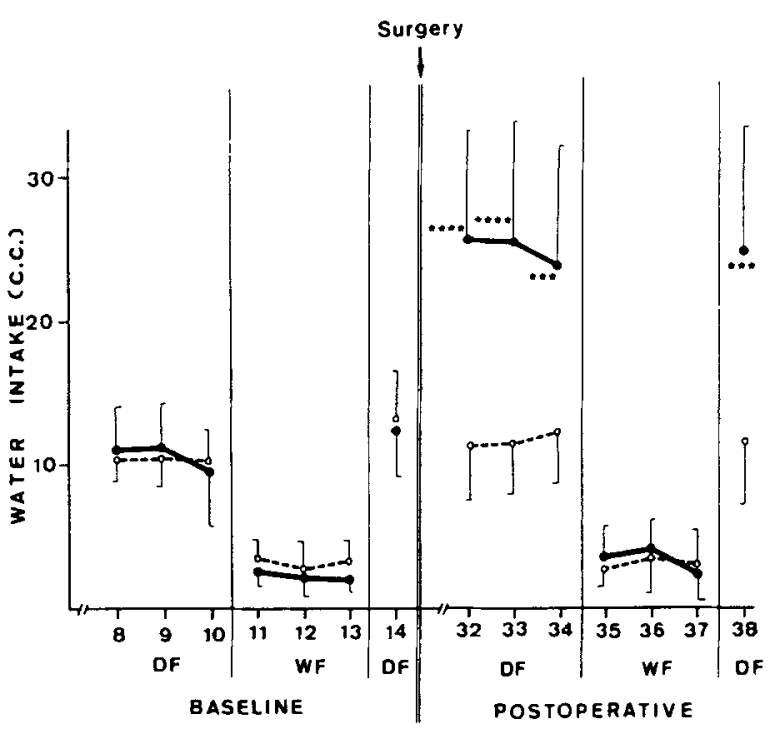

\section{DAYS}

Figure 2. Mean amount of water consumed $( \pm S D)$ in experimental (solid line) and control (dotted line) subjects during the 2-h period of exposure to dry food (DF) and wet food (WF) in the baseline (Days 8-14) and experimental postoperative periods (Days 32-38). DF = dry food; $\mathrm{WF}=$ wet food. Significance level: ${ }^{*} p<.05,{ }^{* *} p<.025$, ${ }^{* * *} p<.01,{ }^{* * * *} p<.001$. Statistical comparisons were performed with Student's $t$ test. Surgery was on Day 14.

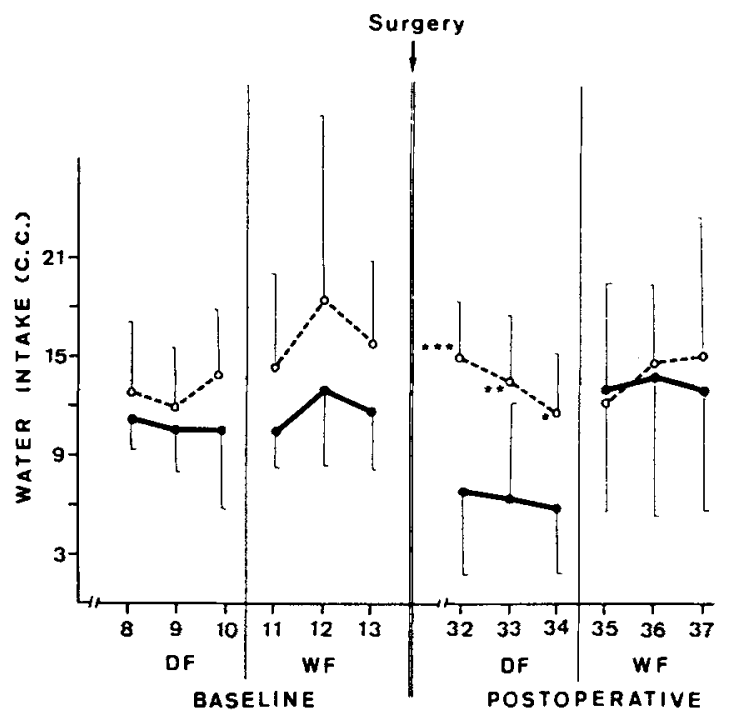

DA Y S

Figure 3. Mean amount of water consumed $( \pm S D)$ in experimental (solid line) and control (dotted line) subjects during the 22-h period of dry-food (DF) and wet-food (WF) deprivation in the baseline (Days 8-13) and experimental postoperative periods (Days 32-37). Water consumed during the 22-h food-deprivation period was not recorded on Days 14 and 38. DF $=$ dry food; WF $=$ wet food. Significance level: ${ }^{*} p<.05,{ }^{* *} p<.025,{ }^{* * *} p<.01,{ }^{* * * *} p<.001$. Statistical comparisons were performed with Student's $t$ test. Surgery was on Day 14. study $[F(1,14)$ dry food $=0.103$, n.s., experimentals $13.9 \mathrm{~g}$ vs. controls $13.8 \mathrm{~g} ; F(1,14)$ wet mash $=3.405$, n.s., experimentals $39.8 \mathrm{~g}$ vs. controls $34.1 \mathrm{~g}$.

\section{DISCUSSION}

The present results show that lesions of the parasympathetic salivary fibers running through the middle ear induce a severe prandial drinking pattern in the rat. The observation that the wet mash diet abolished the anomalous behaviors suggests that these behavioral changes are the consequence of a true deficit in salivary secretion. Moreover, the hypodipsia recorded in the experimental rats throughout the 22-h food-deprivation period suggests that the excess of water consumed by the transected animals during the 2-h period when dry food was presented reflects a failure of nonhomeostatic mechanisms involved in water regulation (e.g., a deficit in salivary secretion) rather than an alteration in a homeostatic system. The excessive intake of water by experimental rats during their 2-h exposure to dry food was, in fact, offset by the minimal amount of water drunk in the following 22-h period, a finding suggesting that homeostatic mechanisms of water regulation were intact and functioning appropriately in these animals.

The salivatory deficit induced in the experimental group most likely affected all three pairs of major salivary glands (parotid, submandibular, and sublingual), as the animals otherwise would not have developed a prandial drinking pattern with polydipsia. Other authors have shown that prandial drinking is only observed following total, but not partial desalivation (Stricker, 1970). Such findings support the notion that intra-auricular surgery effectively transected both the parasympathetic preganglionic fibers to the submandibular-sublingual glands (chorda tympani) and the fibers running to the parotid glands (tympanic nerve).

Moreover, earlier studies in our laboratory provide physiological evidence that surgical intervention as employed here does transect both parasympathetic nerves, that is, the chorda tympani and the tympanic nerve: First, stimulation of the brainstem nucleus parvocellularis (the superior salivary nucleus) evokes submandibularsublingual salivary secretion together with a minimal parotid secretion of parasympathetic nature (Ramos \& Puerto, 1988). Second, this secretion is blocked by the middle ear intervention used in the present report (Ramos, Castillo, \& Puerto, 1989).

The data presented in this article are compatible with previous pharmacological studies that suggest that cholinergic blockade of the three major salivary glands is sufficient to cause prandiality (Chapman \& Epstein, 1970). However, the data obtained with atropine are insufficient to establish a relationship between a specific neuronal system and prandiality. In fact, the intraperitoneal administration of this anticholinergic drug not only blocks parasympathetic secretion of saliva but also affects other neural cholinergic systems involved in buccomandibular responses, including mastication and food 
swallowing (Brodal, 1981). The present experiment is, nonetheless, novel in that it manipulated only the cholinergic control of the salivary glands, by means of parasympathetic denervation. Hence, the findings we present point toward a direct relationship between parasympathetic innervation of the salivary glands and the prandial drinking pattern which characterizes desalivated animals.

\section{REFERENCES}

ABE, K. (1987). Autonomic effects on rat submandibular salivary glands. Journal of Dental Research, 66, 584-585.

ABE, K., N NTTA, H. (1984). Amino acid composition of salivary protein secreted by the parotid glands of rats in response to parasympathomimetic and sympathomimetic drugs. Archives of Oral Biology, 29, 127-130.

Brodal, A. (1981). Neurological Anatomy. New York: Oxford University Press.

Chapman, H. W., \& Epstein, A. N. (1970). Prandial drinking induced by atropine. Physiology \& Behavior, 5, 549-554.

EMMELIN, N. (1987). Nerve interactions in salivary glands. Journal of Dental Research, 66, 509-517.

Epstein, A. N., Spector, D., Samman, A., \& Goldblum, C. (1964).
Severe prandial drinking in the rat without salivary glands. Nature, 201, 1342-1343.

KissilefF, H. R. (1969). Food-associated drinking in the rat. Journal of Comparative \& Physiological Psychology, 67, 284-300

Nicholson, J. E., \& Severin, C. M. (1981). The superior and inferior salivatory nuclei in the rat. Neuroscience Letters, 21, 149-154.

Nomura, S., \& Mizuno, N. (1982). Central distribution of afferent and efferent components of the glossopharyngeal nerve: An HRP study in the cat. Brain Research, 236, 1-13.

PowlEY, T. L. (1977). The ventromedial hypothalamic syndrome, satiety and a cephalic phase hypothesis. Psychological Review, 84, 89-126.

Ramos, J. M. J., \& Puerto, A. (1988). The nucleus parvocellularis reticularis regulates submandibular-sublingual salivary secretion in the rat: A pharmacological study. Journal of the Autonomic Nervous System, 23, 221-228.

Ramos, J. M. J., Castillo, M. E., \& Puerto, A. (1989). Peripheral pathways mediating salivary secretion after nucleus parvocellularis activation in the rat. Brain Research Bulletin, 22, 469-473.

STRICKER, E. M. (1970). Influence of saliva on feeding behavior in the rat. Joumal of Comparative \& Physiological Psychology, 70, 103-112.

Thulin, A. (1976). Motor and secretary effects of nerves on the parotid gland of the rat. Acta Physiologica Scandinavica, 96, 506-511.

(Manuscript received February 21, 1989; revision accepted for publication May 15, 1990.)

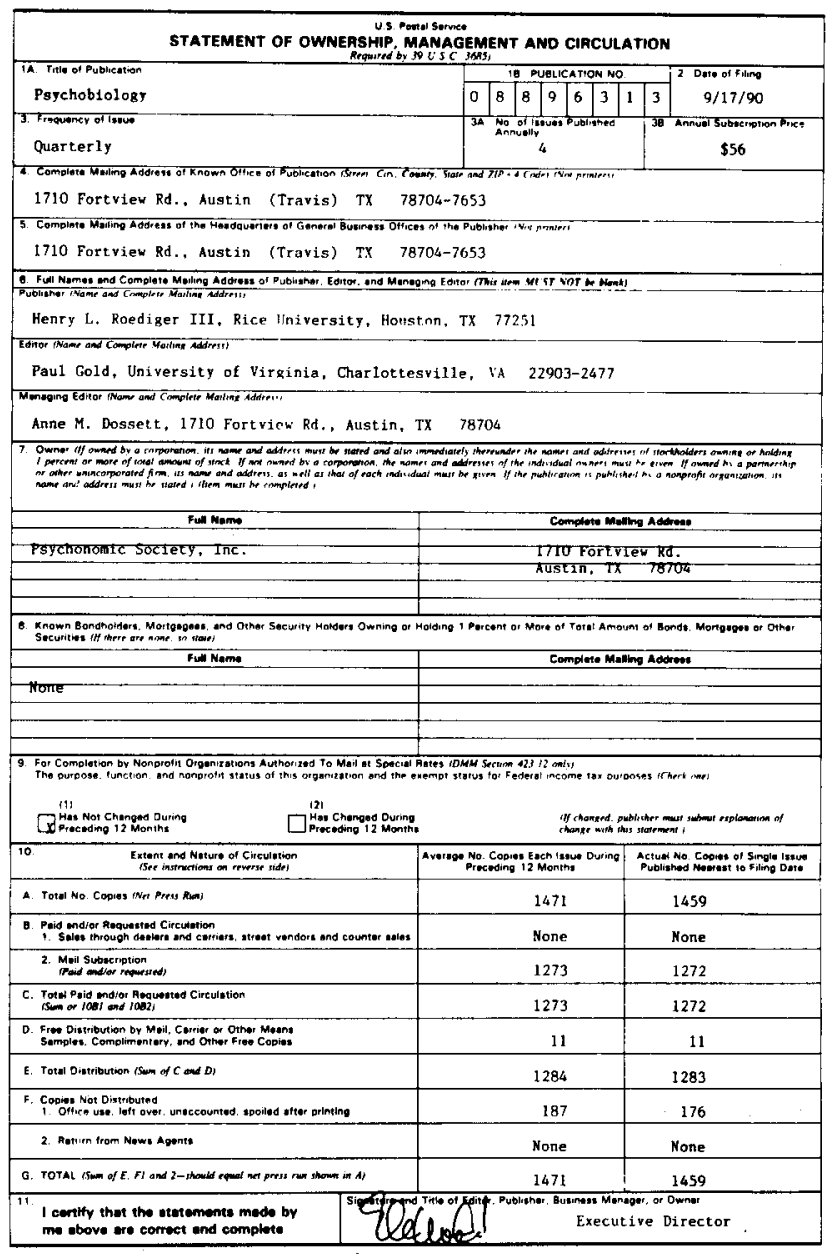

